\title{
Flatness Based Velocity Tracking Control of a Vehicle on a Roller Dynamometer Using a Robotic Driver
}

\author{
Stefan Sailer, Michael Buchholz and Klaus Dietmayer
}

\begin{abstract}
This paper presents a velocity tracking control of a robotic driver that is used for driving industry test cycles on roller dynamometers. As existing commercial robotic drivers show a high complexity, use outdated mechanics and are nevertheless quite expensive, a new robotic driver is developed. The aim is to track a well defined velocity trajectory of a test cycle with high accuracy in an arbitrary vehicle, whereby no learning cycle for determining the specific vehicle behaviour is allowed.

To meet all these conditions, at first a single-wheel model for the longitudinal dynamics of a vehicle is proposed. Additionaly, an approximation for the engine torque is given, enabling the adaption of the resulting model by changing only few parameters. Based on this model, a flatness based velocity tracking control for operating the acceleration pedal is introduced.
\end{abstract}

\section{INTRODUCTION}

During the development phase of new vehicles, many different tests (e.g. climatic, acoustic or emission tests) have to be passed. These tests are usually conducted by means of vehicle dynamometers, composed of rollers on which the test cycles are driven. These vehicle dynamometers simulate the real driving resistance with the result that the measurements are reproducable and can be compared to those of other vehicles. Due to the temporarily very extreme test environment (e.g. high/low temperatures), the development of robotic drivers replacing human drivers was encouraged soon.

Today's commercially available robotic drivers consist of driving mechanics placed on the driver's seat [1]. These mechanics are connected to an overlaid host system that sends positioning commands to the actuators of the robotic driver (i.e. spindle drives) and controls the vehicle in this way along a desired velocity trajectory. Due to the signaling line, the car door or at least the window has to be open, whereby the measurement results of some tests (e.g. electromagnetic compliance (EMC), acoustic, climatic tests) are affected distinctly.

Therefore, a new robotic driver should be developed that obtains the necessary test signals from cognitive abilities. Without additional sensors, the robotic driver will be able to determine the provided pedal force from the actuators (haptic cognition), enabling to mimic human habits especially at gear shifting operations. Moreover, the velocity of the vehicle as well as the engine speed and some other important control

This project is supported by the German Federal Ministry of Economics and Technology (BMWi).

The authors are with the Institute of Measurement, Control an Mircotechnology, Ulm University, 89081 Ulm, Germany \{Stefan.Sailer, Michael.Buchholz, Klaus.Dietmayer $\}$ duni-ulm.de

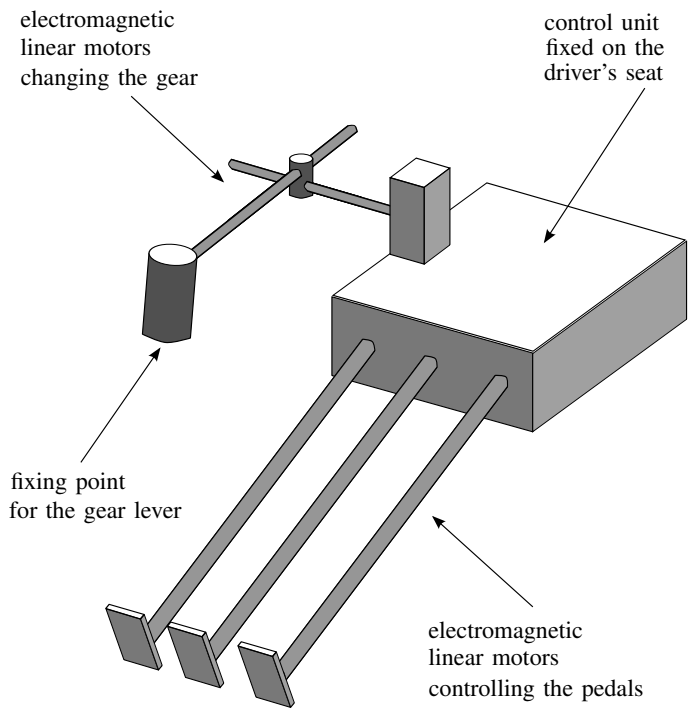

Fig. 1. Sketch of the robotic driver used for driving a vehicle on a roller dynamometer

signals will be determined with other cognitive abilities. Furthermore, the control unit is placed in the vehicles passanger cabin so that a fully autarkic mode of operation will possible.

The proposed robotic driver consists of five electromagnetic linear motors (four for automatic transmission vehicles), enabling a direct linear movement (see Fig. 1). Using two actuators fixed at the gear lever for the rectangular movement of changing the gear, and the other actuators for pushing or releasing the brake pedal, the accelerator, and, if applicable, the clutch pedal, all necessary elements for driving a test cycle can be operated.

The position of each of these actuators is controlled with PID controller. Showing approximatively a proportional closed-loop behaviour without any delay, the dynamics of the actuators will be disregarded in the following.

Apart from the mentioned fully autarkic mode of operation, different driving strategies will be realized, e.g. high accurate driving and fuel-conserving driving, exploiting the allowed tolerance limits of a test cycle.

The challenge of the control design is that the robotic driver must be able to drive any arbitrary vehicle along a desired velocity trajectory, without the need of manually adapting any control parameters offline or driving a learning cycle.

To track a desired velocity trajectory as accurate as possible, several overlaid controllers are necessary, namely a multivariable control for driveaway, a control for decelerating 
the car by pushing the brake, and a control for accelerating and maintaining the speed by operating the acceleration pedal. Only the latter is proposed in this paper.

There have already been considered various velocity control approaches for robotic drivers. A controller based on an $H_{\infty}$-design, as proposed in [2], requires several learning cycles to determine the engine map, the clutch and braking perfomance. [1] proposes a PID controller, though showing a poor accuracy. Furthermore, as they do not use a model based control design, the adaption to different vehicle types must be done intuitively and stability cannot be ensured. As these proposed control approaches do not meet the conditions of universal application to any arbitrary vehicle without driving learning cycles, a flatness based tracking control approach is proposed in this contribution.

This paper is organized as follows. In Section II, a model of a vehicle is introduced that can be adapted to any arbitrary vehicle by changing few parameters. Based on this model, a flatness based control is propesed in Section III, whereby velocity tracking is enabled. First experimental results analyzing the control accuracy and the possibility of obtaining repeatable measurements are shown in Section IV. Finally, in addition to a conclusion drawn in Section V, a short perspective to future work is given.

\section{MODEL OF A VEHICLE}

\section{A. Longitudinal Dynamics}

Deriving a model that describes the longitudinal dynamics of a vehicle has been frequently treated; a detailed example is given in [3]. The most proposed models use many vehicle specific parameters that need to be identified in order to map the dynamics of the vehicle as exactly as possible.

Due to the small number of available measurement signals, most parameters cannot be identified, and usually, they are not known either. As the robotic driver must be universally applicable to any arbitrary vehicle, a model is needed that is as accurate as possible and even can be adapted to the actual vehicle by using a minimum of vehicle specific parameters. The resulting model should describe the most important dynamics of a vehicle, only using parameters that are well know (e.g. engine peak power, vehicle mass) or can be identified easily (e.g. gear ratio).

To achieve this compromise between simplicity and accuracy, several idealizations and simplifying assumptions have to be made. Assuming that the clutch is engaged, all axles are perfectly rigid, and that there is no slip between wheels and road, the longitudinal dynamics of a vehicle can be described as a single-wheel model [4].

This single-wheel model consists of one single inertia $I_{t o t}$ rotating with the engine speed $\omega_{e}$ and containing all rotating components of the drivetrain $I_{r o t}$ and the vehicle inertia, whereby the intertias and torques at the load side of the gearbox are lumped with the inertias and torques of the engine-side of the gearbox:

$$
I_{\text {tot }}=I_{\text {rot }}\left(i_{\text {tot }}\right)+\frac{m_{v} r_{d y n}^{2}}{i_{\text {tot }}^{2}},
$$

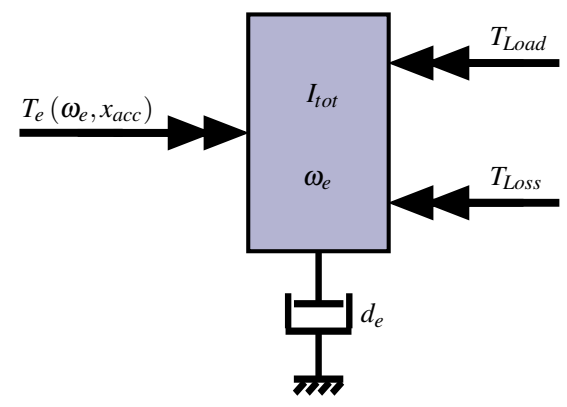

Fig. 2. Structure of resulting single-wheel model

where $m_{v}$ denotes the vehicle mass, $r_{d y n}$ is the dynamic wheel radius and $i_{t o t}$ is the total gear transmission ratio of the drivetrain.

Acceleration of this inertia is realized by the engine torque $T_{e}$, which depends on the position of the acceleration pedal $x_{a c c}$ and the actual engine speed. The viscous damping $d_{e}$ of the engine, the load torque $T_{\text {load }}$ resulting at the engine-side of the gearbox, and the torque $T_{\text {loss }}$ containing the losses of the drivetrain work against the accelerating engine torque (see Fig. 2):

$$
I_{\text {tot }} \dot{\omega}_{e}=T_{e}\left(x_{\text {acc }}, \omega_{e}\right)-d_{e} \omega_{e}-T_{\text {loss }}-\frac{1}{i_{\text {tot }}} T_{\text {load }}
$$

Furthermore, the vehicle will drive only on a dynamometer, therefore the grade resistance can be ignored and the resulting load torque only consists of the air $\operatorname{drag} F_{a}$ and the rolling resistance $F_{r}$ :

$$
T_{\text {load }}=r_{d y n} \cdot\left(F_{a}+F_{r}\right)=r_{d y n} \cdot\left(\frac{1}{2} \rho c_{d} A v^{2}+c_{r r} m_{\nu} \mathrm{g}\right),
$$

where $\rho$ denotes the density of air, $c_{d}$ is the aerodynamic drag coefficient, $A$ is the cross-sectional area, $c_{r r}$ is the rolling resistance coefficient and $v$ is the velocity of the vehicle, which can be expressed as a function of engine speed:

$$
v=\frac{r_{d y n}}{i_{\text {tot }}} \cdot \omega_{e}:=\frac{1}{\gamma} \cdot \omega_{e} .
$$

As the resistance forces are simulated by the roller dynamometer, the parameters used in (3), except $r_{d y n}$, can be considered as known. For $d_{e}, r_{d y n}, i_{t o t}$, and $I_{r o t}$, values of a common mid-range car given in the literatur are used (e.g. [5], [6]). Considering several performance charts of different engines and determining the mean value of the losses in the drivetrain, $T_{l o s s}$ will be assumed to be $20 \%$ of the engine torque at peak power.

\section{B. Engine Map}

In order to obtain a complete model of a vehicle that relates the position of the acceleration pedal to the longitudinal dynamics, it is necessary to find a relation between the engine torque and the position of the acceleration pedal. There are several models for combustion engines proposed in literature (e.g. [5], [7]), which have been derived by applying the thermodynamic laws and considering the engine design. 
TABLE I

COEFFICIENTS OF THE POLYNOMIAL MODEL FOR THE FULL-LOAD CURVE OF DIFFERENT ENGINE TYPES PROPOSED IN [8].

\begin{tabular}{lccc}
\hline engine type & $C_{1}$ & $C_{2}$ & $C_{3}$ \\
\hline spark ignition & $\frac{P_{\max }}{\omega_{P, \max }}$ & $\frac{P_{\max }}{\omega_{P, \max }^{2}}$ & $-\frac{P_{\max }}{\omega_{P, \max }^{3}}$ \\
indirect injection diesel & $0.6 \frac{P_{\max }}{\omega_{P, \max }}$ & $1.4 \frac{P_{\max }}{\omega_{P, \max }^{2}}$ & $-\frac{P_{\max }}{\omega_{P, \max }^{3}}$ \\
direct injection diesel & $0.87 \frac{P_{\max }}{\omega_{P, \max }}$ & $1.13 \frac{P_{\max }}{\omega_{P, \max }^{2}}$ & $-\frac{P_{\max }}{\omega_{P, \max }^{3}}$ \\
\hline
\end{tabular}

As these models rely on too many propietary parameters and inaccessible test signals, they are inapplicable for our application.

Using the empirical fact that the variation of the engine torque is much larger at the beginning of the acceleration pedal travel than at the end, the following approximation will be used instead:

$$
T_{e}\left(x_{a c c}, \omega_{e}\right)=T_{F L}\left(\omega_{e}\right) \cdot \sqrt{\frac{x_{a c c}}{x_{a c c, \max }}},
$$

where $x_{a c c, \text { max }}$ denotes the end of the acceleration pedal travel and $T_{F L}\left(\omega_{e}\right)$ is the engine torque at $x_{a c c, \max }$.

\section{Full-load Curve}

Because of the assumption (5) made for the engine map, it is necessary to find an approximation for the rotation speed dependent torque $T_{F L}\left(\omega_{e}\right)$ that is also known as the full-load curve of the engine. This approximation is to be independent of proprietary parameters and may only rely on the known stated engine peak power $P_{\max }$, the associated engine speed $\omega_{P, \max }$, and the corresponding engine torque $T_{P, \max }=\frac{P_{\max }}{\omega_{P, \max }}$. In [8], a polynomial model is proposed that meets these requirements:

$$
T_{F L}\left(\omega_{e}\right)=\sum_{i=1}^{3} C_{i} \omega^{i-1},
$$

where the coefficients are given in Table I. As this model is quite inaccurate especially at low engine speeds (see Fig. 3), a new approximation will be proposed.

At first, the engine speed range is split up into three parts: the first from $\omega_{1000}=\frac{\pi}{30} 1000 \frac{\mathrm{rad}}{\mathrm{s}}$ to $\omega_{1500}=\frac{\pi}{30} 1500 \frac{\mathrm{rad}}{\mathrm{s}}$, the second from $\omega_{1500}$ to the engine speed at peak torque $\omega_{T, \max }$, and the third from $\omega_{T, \max }$ to $\omega_{P, \max }$. Then, the full-load curve can be approximated in every range as a second order polynomial:

$$
T_{F L}\left(\omega_{e}\right)=\xi_{1}\left(\omega_{e}\right) \cdot \omega_{e}^{2}+\xi_{2}\left(\omega_{e}\right) \cdot \omega_{e}+\xi_{3}\left(\omega_{e}\right),
$$

where the coefficients $\xi_{i}\left(\omega_{e}\right), i=1, \ldots, 3$ generally differ in each engine speed range. To determine the coefficents of these polynomials, the resulting torque curve must go through the torque at peak power $T_{P, \max }$, the peak torque $T_{\max }$ at $\omega_{T, \max }$ and the full-load torques $T_{1500}$ and $T_{1000}$ at $\omega_{1500}$ and $\omega_{1000}$. The unknown values for $T_{\max }, T_{1500}, T_{1000}$ and $\omega_{M, \max }$ can be calculated from $T_{P, \max }$ and $\omega_{P, \max }$ by means of the ratios given in Table II. Considering several full-load
TABLE II

RATIOS TO DETERMINE THE CHARACTERISTIC POINTS OF A FULL-LOAD CURVE

\begin{tabular}{lcccc}
\hline engine type & $T_{P, \max }$ & $\frac{T_{P, \max }}{T_{1000}}$ & $\frac{T_{P, \max }}{T_{1500}}$ & $\frac{\omega_{P, \max }}{T_{T, \max }}$ \\
\hline spark ignition & 1.273 & 1.095 & 0.881 & 1.706 \\
diesel & 1.503 & 0.882 & 0.785 & 2.016 \\
\hline
\end{tabular}

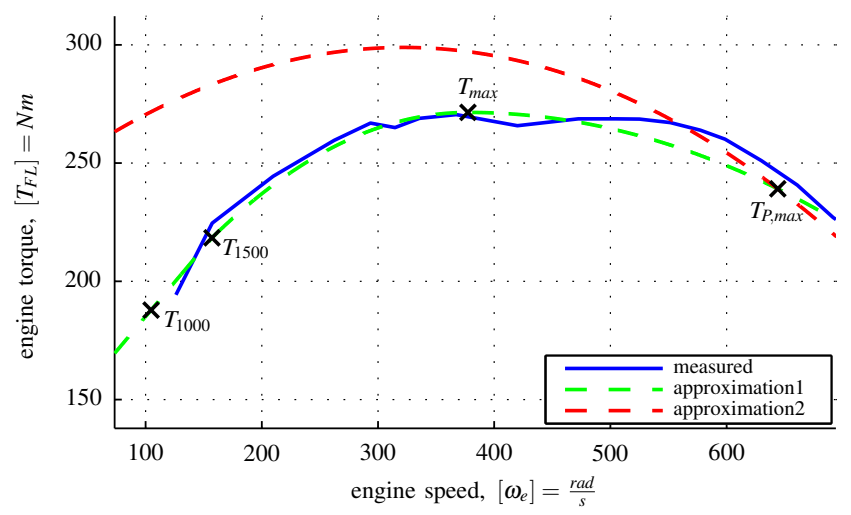

(a) Audi A6 Avant 2.8 FSI -07 (stated peak power: 154kw@6150rpm)

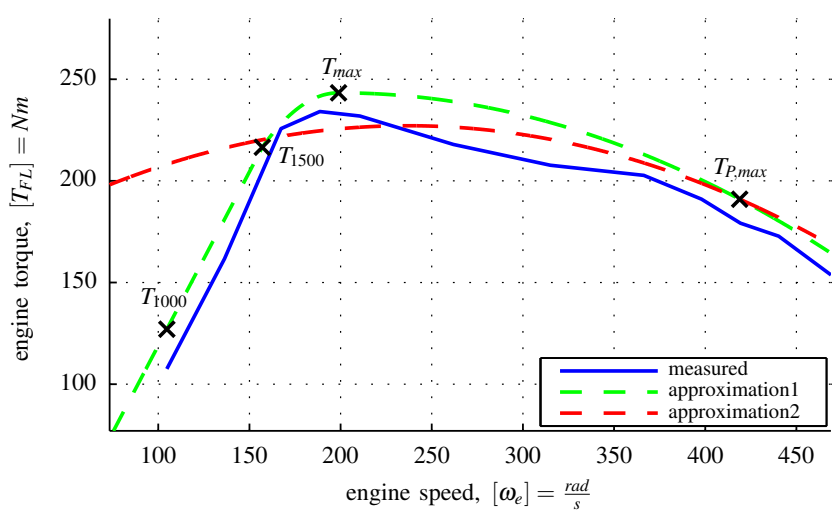

(b) Volvo V50 1.6DRIVe -09 (stated peak power: 80kw@4000rpm)

Fig. 3. Full-load curves of a spark ignition engine (top) and a direct injection diesel engine (bottom): approximation 1 by (7) proposed in this paper, approximation 2 by (6) according to [8]

curves of different engines, in a first approximation these ratios are assumed to be constant, distinguishing between spark ignition and diesel engines. Thus, the values given in Table II are determined by averaging.

To avoid that the system of equations for determining the coefficients of the polynomial (7) is under-determined, differentiability at the boundaries between the speed ranges must be ensured.

The results of this new approximation in comparison with approximation proposed in [8] are illustrated for two different cars exemplary in Fig. 3. It can be seen that the shapes of the new approximation and the measured curves correspond quite well, but due to the inconsistency between stated and measured engine peak power, an offset exists. 


\section{FLATNESS BASED VELOCITY TRACKING CONTROL}

\section{A. Differential Flatness}

The terminology differential flatness was introduced in [9] and enabled a new way to design a tracking controller for a nonlinear flat system

$$
\dot{\mathbf{x}}=\mathbf{f}(\mathbf{x}, u, t), \quad \mathbf{x}\left(t_{0}\right)=\mathbf{x}_{0}
$$

with the states $\mathbf{x} \in \mathbb{R}^{n}$ and the input $u \in \mathbb{R}$. The system is differentially flat, if it has a flat (possibly fictive) output $y_{f}$ that meets the following conditions:

- The flat output must be a function of the states:

$$
y_{f}=\Phi(\mathbf{x}) \text {. }
$$

- Both, the states and the input must be a function of the flat output and its time derivatives:

$$
\begin{aligned}
& \mathbf{x}=\psi_{x}\left(y_{f}, \dot{y}_{f}, \ldots, y_{f}^{(n-1)}\right), \\
& u=\psi_{u}\left(y_{f}, \dot{y}_{f}, \ldots, y_{f}^{(n)}\right) .
\end{aligned}
$$

Equations (10) and (11) are called differential state and differential input parameterizations, respectively. Note that these parameterizations describe the inverse system of (8) for the flat output, so that the non-linearities of the system can be linearized exactly. Hence, all dynamics of a flat system can be described by the flat output and its time derivatives, too.

Defining the engine speed $\omega_{e}$ as a state and the relative position of the acceleration pedal $\frac{x_{a c c}}{x_{a c c, \max }}$ as the input, the velocity $v$ of the vehicle is a flat output of the system using (2)-(5) and (7) for the dynamics of the vehicle. This yields to the parameterization of the state

$$
\psi_{x}=\gamma \cdot v
$$

and the parameterization of the input

$$
\psi_{u}=\left[\frac{I_{t o t} \gamma \dot{v}+d_{e} \gamma \nu+\frac{r_{d y n}}{i_{t o t}}\left(\frac{1}{2} \rho c_{d} A v^{2}+c_{r r} m_{v} \mathrm{~g}\right)}{\xi_{1}(\gamma v) \gamma^{2} v^{2}+\xi_{2}(\gamma v) \gamma v+\xi_{3}(\gamma v)}\right]^{2} .
$$

\section{B. Flatness based Feedforward Control}

If a given system (8) meets the condition of flatness, the design of an open-loop control is straightforward. Substituting in the parameterizations (10) and (11) the flat output and its time derivatives by a sufficiently smooth desired trajectory $y_{f, d}(t) \in \mathcal{C}^{n}$ provides the control $u_{d}(t)$ and the corresponding state trajectory $\mathbf{x}_{d}(t)$ [9]:

$$
\begin{aligned}
u_{d} & =\psi_{u}\left(y_{f, d}, \dot{y}_{f, d}, \ldots, y_{f, d}^{(n)}\right) \\
& =\left[\frac{I_{t o t} \gamma \dot{v}_{d}+d_{e} \gamma v_{d}+\frac{r_{d y n}}{i_{t o t}}\left(\frac{1}{2} \rho c_{d} A v_{d}^{2}+c_{r r} m_{v} \mathrm{~g}\right)}{\xi_{1}\left(\gamma v_{d}\right) \gamma^{2} v_{d}^{2}+\xi_{2}\left(\gamma v_{d}\right) \gamma v_{d}+\xi_{3}\left(\gamma v_{d}\right)}\right]^{2}, \\
\mathbf{x}_{d} & =\psi_{x}\left(y_{f, d}, \dot{y}_{f, d}, \ldots, y_{f, d}^{(n-1)}\right) \\
& =\gamma \cdot v_{d},
\end{aligned}
$$

where the set $\mathcal{C}^{n}$ contains all differentiable functions $f$ whose derivatives $\dot{f}, \ddot{f}, \ldots, f^{(n)}$ exist and are continuous.
As the velocity trajectories of many emission test cycles are not differentiable, the desired velocity trajectories have to be smoothed. The highest time derivative of the velocity occuring in the control trajectory (14) is the acceleration $\dot{v}_{d}$, thus a first-order lag element is used as a lowpass filter to obtain a smooth velocity trajectory.

Note that the feedforward control (14) only steers the system along the desired output trajectory if the model of the system maps the dynamics accurately enough, no disturbances influence the plant, and if the real inital condition and the initial condition resulting from the paramterization (15) are consistent:

$$
\begin{aligned}
\mathbf{x}\left(t_{0}\right) & =\psi_{x}\left(y_{f, d}\left(t_{0}\right), \dot{y}_{f, d}\left(t_{0}\right), \ldots, y_{f, d}^{(n-1)}\left(t_{0}\right)\right), \\
\Leftrightarrow v\left(t_{0}\right) & =v_{d}\left(t_{0}\right)
\end{aligned}
$$

\section{Flatness based Tracking Control}

Because of the simplifying assumptions and approximations made in Section II, the flatness based feedforward control (14) is insufficiently accurate, resulting in a distinct divergence between desired and actual velocity trajectory. Thus, a tracking control based on state feedback is designed that stabilizes the tracking of the desired trajectory ([10], [11]).

For this pupose, the nonlinear flat system (8) is linearized by applying a state dependent input transformation.

Inserting the new input

$$
\hat{u}=y_{f}^{(n)}
$$

and the new state

$$
\mathbf{z}=\left[z_{1}, z_{2}, \ldots, z_{n}\right]^{T}=\left[y_{f}, \dot{y}_{f}, \ldots, y_{f}^{(n-1)}\right]^{T}
$$

into (11) yields to the following parameterization of the input:

$$
u=\psi_{u}\left(z_{1}, z_{2}, \ldots, z_{n}, \hat{u}\right) .
$$

By means of this linearizing input transformation, the nonlinear system can be described as a chain of integrators of the length $n$, with the new input $\hat{u}$ and the output $y_{f}$, i.e. the Brunovský normal form:

$$
\begin{aligned}
\dot{z}_{i} & =z_{i+1}, \quad i=1, \ldots, n-1, \\
\dot{z}_{n} & =\hat{u} .
\end{aligned}
$$

In order to design a tracking control for this linear chain of integrators, the tracking error is defined as follows:

$$
e=y_{f}-y_{f, d}=z_{1}-z_{1, d} .
$$

To force a desired transient behaviour of the tracking error, the differential equation

$$
e^{(n)}+p_{n-1} e^{(n-1)}+\ldots+p_{0} e=0
$$

is considered. The dynamic coefficients $p_{i}$ in (23) are determined by equating the coefficients of the characteristical 
polynomial of the tracking error with the coefficients of the desired dynamic defined by the eigenvalues $\lambda_{d, i}$ :

$$
\lambda^{n}+p_{n-1} \lambda^{n-1}+\ldots+p_{0} \stackrel{!}{=} \prod_{j=0}^{n}\left(\lambda-\lambda_{j, d}\right) .
$$

Using (20)-(22) in (23) yields to

$$
\hat{u}=\dot{z}_{n, d}+\sum_{i=1}^{n-1} p_{i}\left(z_{i+1}-z_{i+1, d}\right) .
$$

This expression for the new input $\hat{u}$ takes the desired transient behaviour of the tracking error into account. Inserting (25) in the parameterization for the input (19), yields the feedback law

$$
u=\psi_{u}\left(\mathbf{z}, \mathbf{z}_{d}\right)=\psi_{u}\left(z_{1}, z_{2}, \ldots, z_{n}, z_{1, d}, z_{2, d}, \ldots, z_{n, d}\right) .
$$

Due to parameter uncertainties, inaccuracies of the model or disturbances, it is common to add an integral summand to (25):

$$
\hat{u}=\dot{z}_{n, d}+\sum_{i=1}^{n-1} p_{i}\left(z_{i+1}-z_{i+1, d}\right)+p_{-1} \int\left(z_{1}-z_{1, d}\right) \mathrm{d} t .
$$

Then, the extended characteristic polynomial has to be considered to determine the dynamic coefficients $p_{i}$ :

$$
\lambda^{n+1}+p_{n-1} \lambda^{n}+\ldots+p_{0} \lambda+p_{-1} \stackrel{!}{=} \prod_{j=0}^{n+1}\left(\lambda-\lambda_{j, d}\right) .
$$

Using again the velocity $v$ of the vehicle as flat output of the system derived in Section II, the feedback law (26) provides

$$
u=\left[\frac{I_{t o t} \gamma \hat{u}+d_{e} \gamma v+\frac{r_{d y n}}{i_{t o t}}\left(\frac{1}{2} \rho c_{d} A v^{2}+c_{r r} m_{v} \mathrm{~g}\right)}{\xi_{1}(\gamma v) \gamma^{2} v^{2}+\xi_{2}(\gamma v) \gamma v+\xi_{3}(\gamma v)}\right]^{2}
$$

with the new input

$$
\hat{u}=\dot{v}_{d}+p_{0}\left(v-v_{d}\right)+p_{-1} \int\left(v-v_{d}\right) \mathrm{d} t .
$$

\section{EXPERIMENTAL RESULTS}

Driving first test cycles on the roller dynamometer with the robotic driver and the velocity tracking control proposed in Section III-C, two important properties were analyzed. On the one hand, the accuracy and the robustness of the controller was determined, and, on the other hand, the repeatability of measurement results was investigated.

\section{A. Control Accuracy and Robustness}

For determining the accuracy and the robustness of the proposed controller, different test cycles (e.g. ECE Urban Driving Cycles (UDC), Extra Urban Driving Cycle (EUDC), Federal Test Procedure (FTP-75), (see [12], [13]) were driven with different vehicles. The feedback law (29) was adapted for the different cars by using the few stated parameters from Section II (e.g. engine peak power with associated engine speed, vehicle mass), whereby the dynamic parameters $p_{i}$ in (30) remained constant.

The achieved results were always quite similar with respect to the control accuracy, so that the robustness of the velocity control proposed in Section III-C is given. Fig. 4
TABLE III

RESULTS OF THE REPEATABILITY TEST: RELATED ENERGY, EMITTED CARBON DIOXIDE AND COVERED DISTANCE WITH CORRESPONDING COEFFICENTS OF VARIATIONS

\begin{tabular}{cccc}
\hline cycle no. & energy $[\mathrm{kJ}]$ & emitted $\mathrm{CO}_{2}[\mathrm{~g}]$ & distance $[\mathrm{m}]$ \\
\hline 1 & 3788.39 & 2482 & 12353.6 \\
2 & 3789.03 & 2477 & 12354.5 \\
3 & 3788.04 & 2473 & 12354.4 \\
4 & 3789.03 & 2481 & 12357.0 \\
5 & 3787.34 & 2482 & 12356.7 \\
\hline $\mathrm{CV}$ & $0.019 \%$ & $0.159 \%$ & $0.012 \%$ \\
\hline
\end{tabular}

illustrates exemplary measurements of a part of the UDC, driven with a diesel engined vehicle (97kW@ 4400rpm). The reference and the actual velocity trajectory are depicted as well as the corresponding deviation with the allowed tolerance limits of $\pm 2 \frac{\mathrm{km}}{\mathrm{h}}$ and the relative position of the acceleration pedal.

The largest deviations occur during the moments when the tracking controller is not active, i.e. driveaway followed by the short distance driven in the first gear, shifting up or down a gear and decelerating the vehicle by applying the brake. When the clutch is engaged again after changing the gear, the proposed control for the acceleration pedal is reactivated. At these moments, the asymtotic stable behaviour of the tracking error and the good velocity tracking behaviour can be seen. Due to the loss of speed during shifting up a gear, the position of the acceleration pedal increases strongly to accelerate the vehicle fast to get back onto the reference trajectory. This behaviour ensures on this way a good control accuracy.

\section{B. Repeatable Driving}

Examining, if using the robotic driver can produce repeatable measurements, the same test cycle was driven five times with the same vehicle under similar conditions. This fifteen minutes lasting cycle was composed of the UDC, the EUDC and the first 300 seconds of the FTP-75. For each of the five driven cycles, the released energy from the vehicle to the roller dynamometer, the emitted carbon dioxide $\left(\mathrm{CO}_{2}\right)$, and the distance covered by the vehicle have been measured. The results of the three measurands with the corresponding coefficents of variation are given in Table III. Note that the the coefficents of variation average out only few per mille. Comparing these results against those of an experienced human test driver whose coefficents of variation for emitted $\mathrm{CO}_{2}$ is about three percent, the possibility of repeatable driving with the robotic driver can clearly be considered as possible.

\section{CONCLUSIONS AND FUTURE WORK}

\section{A. Conclusions}

In this paper, a nonlinear model for arbitrary vehicles with combustion engines was proposed. The torque of the combustion engine has been approximated as a function of 

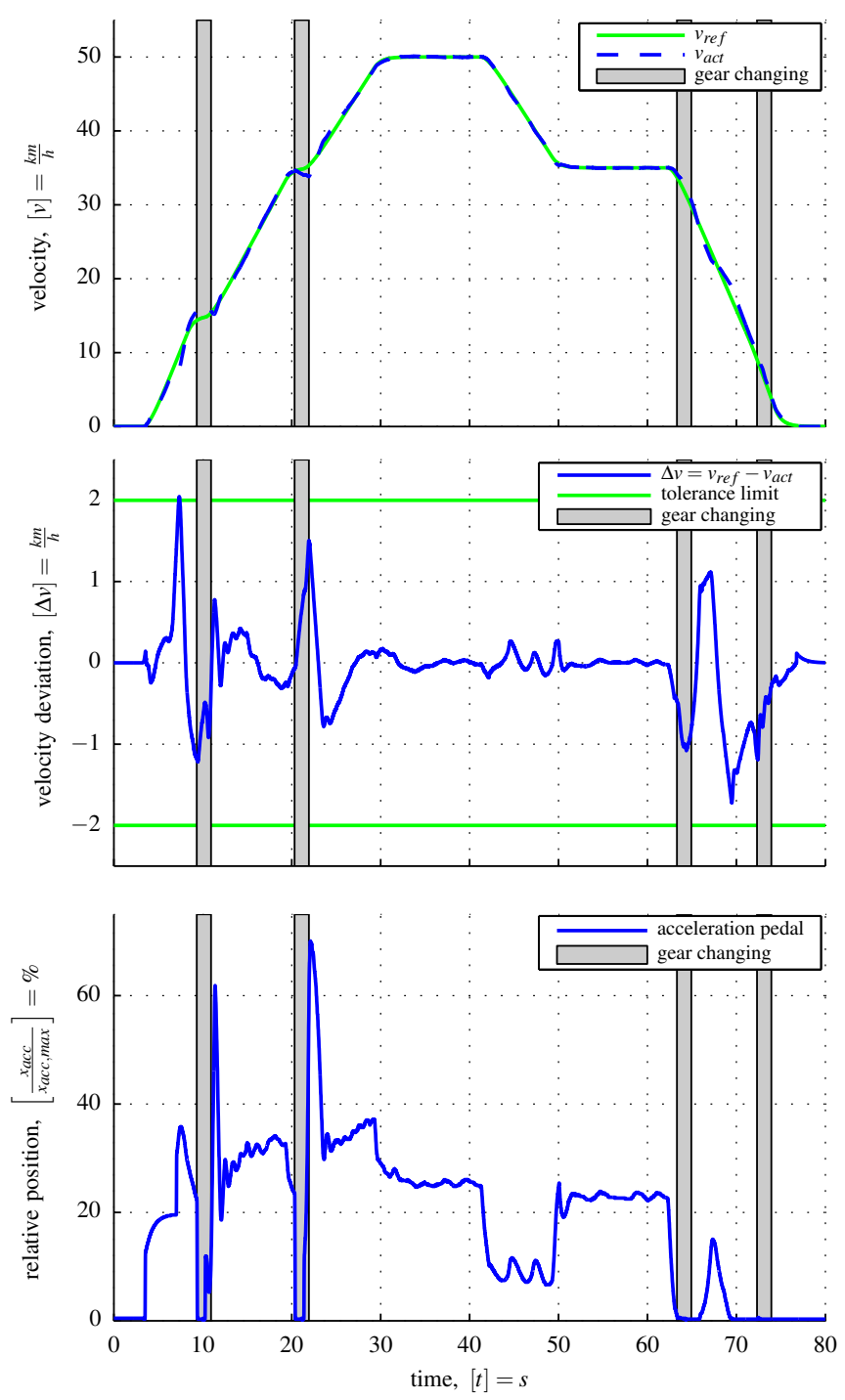

Fig. 4. Part of the UDC, grey rectangles mark the time span for changing the gear. From top to bottom: actual and desired velocity of the vehicle, speed difference as control deviation with allowed tolerance limits $\left( \pm 2 \frac{\mathrm{km}}{\mathrm{h}}\right)$, relative position of the accelerator pedal

the engine speed and the relative position of the acceleration pedal, only knowing the maximal engine power and the related engine speed.

Moderate complexity allows to adjust the model to different vehicles by changing only few parameters, which are known in advance or can be identified during the test cycle. For all the simplifications made, the model of the powertrain takes into account the most important dynamics of a vehicle and is a good basis for the proposed flatness based velocity tracking controller of the acceleration pedal.

First tests did not only show a good accuracy and a satisfying tracking behaviour of the control, but also a good robustness against uncertanties of parameters, and simplifications of the model. Furthermore, the possibility of repeatable driving using the robotic driver could be proved.

\section{B. Future Works}

One important next step is to identify the unknown vehicle parameters (e.g. ratio, dynamic wheel radius, inertias) from the measured signals to obtain a more accurate model. But as the approximated engine torque involves the largest model inaccuracies, an online adaption of this approximation will be indispensable. With the adapted engine map and the identified parameters a better feedforward behaviour of the controller should be achieved.

Based on this controller, a fuel-conserving driving stategy exploitating the allowed tolerance limits can be developed.

\section{REFERENCES}

[1] H. Namik, T. Inamura, and K. Stol, "Development of a Robotic Driver for Vehicle Dynamometer Testing," in Proceedings of the Australasian Conference on Robotics and Automation, University of Auckland, New Zealand, December 6-8 2006.

[2] K. Müller and W. Leonhard, "Computer Control of a Robotic Driver for Emission Tests," in Proceedings of the 1992 International Conference on Industrial Electronics, Control, Instrumentation, and Automation, vol. 3, 1992, pp. 1506 - 1511.

[3] F. Vasca, L. Iannelli, A. Senatore, and M. Scafati, "Modeling Torque Transmissibility for Automotive Dry Clutch Engagement," in American Control Conference, 2008, pp. 306 - 311.

[4] P. Wältermann, "Modelling and Control of the Longitudinal and Lateral Dynamics of a Series Hybrid Vehicle," in Proceedings of the 1996 IEEE International Conference on Control Applications, Sep. 1996, pp. $191-198$.

[5] D. H. McMahon and J. K. Hedrick, "Longitudinal Model Development For Automated Roadway Vehicles," Institute of Transportation Studies, UC Berkeley, Institute of Transportation Studies, Research Reports, Working Papers, Proceedings 102828, Jan. 1990. [Online]. Available: http://ideas.repec.org/p/cdl/itsrrp/102828.html

[6] U. Kramer, Kraftfahrzeugführung: Modelle - Simulation - Regelung. Hanser Fachbuch, 2007.

[7] D. J. Dobner and R. D. Fruechte, "An Engine Model for Dynamic Engine Control Development," in American Control Conference, 1983, 1983, pp. $73-78$.

[8] G. Genta, Motor Vehicle Dynamics: Modeling and Simulation. World Scientific Publishing Co. Pte. Ltd., 1997.

[9] M. Fliess, J. Lévine, and P. Rouchon, "Flatness and Defect of Nonlinear Systems: Introductory Theory and Examples," International Journal of Control, vol. 61, pp. 1327-1361, 1995.

[10] M. Kletting, E. Hofer, and F. Antritter, "Guaranteed Robust Tracking with Flatness Based Controllers Applying Interval Methods," in 12th GAMM - IMACS International Symposium on Scientific Computing, Computer Arithmetic and Validated Numerics, 2006.

[11] A. Hildebrandt, O. Sawodny, R. Neumann, and A. Hartmann, "A Flatness Based Design for Tracking Control of Pneumatic Muscle Actuators," in 7th International Conference on Control, Automation, Robotics and Vision, vol. 3, 2002, pp. 1156 - 1161.

[12] European Economic Community, "Council Directive 70/220/EEC on the Approximation of the Laws of the Member States on Measures to be Taken Against Air Pollution by Emissions From Motor Vehicles," 1970.

[13] Code of Federal Regulations, "Title 40: Protection of Environment - Chapter I: Environmental Protection Agency - Part 86: Control of Emissions From New and In-Use Highway Vehicles and Engines," 1992. 